\title{
Genes that encodes NAGT, MIF1 and MIF2 are not virulence factors for kala-azar caused by Leishmania infantum
}

\author{
Bruno Guedes Alcoforado Aguiar ${ }^{[1]}$, Daniela Lemos Coelho ${ }^{[1]}$, Dorcas Lamounier Costa ${ }^{[1]}$, \\ Betânia Paiva Drumond ${ }^{[2]}$, Luiz Felipe Leomil Coelho ${ }^{[3]}$, Lívio Carvalho Figueiredo ${ }^{[4]}$, \\ Danielle Alves Zacarias ${ }^{[1]}$, Jailthon Carlos da Silva ${ }^{[1]}$, Diego Peres Alonso ${ }^{[5]}$, \\ Paulo Eduardo Martins Ribolla ${ }^{[5]}$, Edna Aoba Yassui Ishikawa ${ }^{[6]}$, \\ Samara Belchior Gaído ${ }^{[1]}$ and Carlos Henrique Nery Costa ${ }^{[1]}$
}

[1]. Laboratório de Leishmanioses, Instituto de Doenças Tropicais Natan Portella, Universidade Federal do Piauí, Teresina, PI. [2]. Laboratório de Virologia, Universidade Federal de Juiz de Fora, Juiz de Fora, MG, Brasil.. [3]. Laboratório de Vacinas, Universidade Federal de Alfenas, Alfenas, MG. [4]. Departamento de Agricultura, Centro de Ciências Humanas, Sociais e Agrárias, Universidade Federal da Paraíba, Bananeiras, PB. [5]. Departamento de Parasitologia, Instituto de Biociências, Universidade Estadual Paulista, São Paulo, SP. [6]. Laboratório de Biologia Molecular, Núcleo de Medicina Tropical, Universidade Federal do Pará, Belém, PA.

\begin{abstract}
Introduction: Kala-azar is a disease resulting from infection by Leishmania donovani and Leishmania infantum. Most patients with the disease exhibit prolonged fever, wasting, anemia and hepatosplenomegaly without complications. However, some patients develop severe disease with hemorrhagic manifestations, bacterial infections, jaundice, and edema dyspnea, among other symptoms, followed by death. Among the parasite molecules that might influence the disease severity are the macrophage migration inhibitory factor-like proteins (MIF1 and MIF2) and N-acetylglucosamine-1-phosphotransferase (NAGT), which act in the first step of protein N-glycosylation. This study aimed to determine whether MIF1, MIF2 and NAGT are virulence factors for severe kala-azar. Methods: To determine the parasite genotype in kala-azar patients from Northeastern Brazil, we sequenced the NAGT genes of $L$. infantum from 68 patients as well as the MIF1 and MIF2 genes from 76 different subjects with diverse clinical manifestations. After polymerase chain reaction (PCR), the fragments were sequenced, followed by polymorphism identification. Results: The nucleotide sequencing of the 144 amplicons revealed the absence of genetic variability of the NAGT, MIF1 and MIF2 genes between the isolates. The conservation of these genes suggests that the clinical variability of kala-azar does not depend upon these genes. Additionally, this conservation suggests that these genes may be critical for parasite survival. Conclusions: NAGT, MIF1 and MIF2 do not alter the severity of kala-azar. NAGT, MIF1 and MIF2 are highly conserved among different isolates of identical species and exhibit potential for use in phylogenetic inferences or molecular diagnosis.
\end{abstract}

Keywords: Genetic diversity. Kala-azar. Visceral leishmaniasis. Macrophage inhibition factor. Tropical diseases.

Single nucleotide polymorphism.

\section{INTRODUCTION}

Leishmaniasis is a group of diseases that compromise skin, mucous membranes and visceral organs. Leishmaniasis diseases are zoonotic, widely distributed globally and are closely associated with poverty ${ }^{2}$. As such, leishmaniasis is an important public health problem ${ }^{1}$. The annual incidence is estimated to be approximately two million people, and approximately 350 million people are at risk of contracting the disease ${ }^{2,3}$. Among the types of leishmaniasis, kala-azar is well known and is considered

\footnotetext{
Address to: Dr. Carlos Henrique Nery Costa. Laboratório de Leishmanioses/ Instituto de Doenças Tropicais Natan Portella/UFPI. Rua Artur de Vasconcelos 151 Sul, 64001-450 Teresina, PI, Brasil.

Phone: 5586 3221-3413; Fax: 5586 3222-3248

e-mail: chncosta@gmail.com; onurbguedes@gmail.com

Received 11 August 2014

Accepted 13 October 2014
}

by the World Health Organization (WHO) to be one of the six most significant endemic diseases in the World.

The majority of infected individuals are asymptomatic ${ }^{4-6}$; however, visceral leishmaniasis (VL) exhibits clinical and laboratory presentations that include a prolonged course of fever, pallor, weight loss, hepatosplenomegaly and pancytopenia ${ }^{7}$. With these classic manifestations, clinical presentations including bleeding and bacterial infections are present in the most severe forms of the disease and are associated with mortality ${ }^{8}$.

The identification of genetic variants is critical for the detection of a predisposition to various diseases ${ }^{9}$. High conservation of genes suggests that a small number of speciesspecific genes are relevant to pathogenicity or virulence. Leishmania, the etiological agent of kala-azar, has 34-36 chromosomes. From a total of approximately 8,300 genes in each Leishmania species, slightly more than 200 are differentially distributed between the genomes of Leishmania infantum, Leishmania major and Leishmania braziliensis. The most divergent species is $L$. braziliensis, containing 49 different 
genes, followed by $L$. infantum, with 27 genes specific to the species; the least divergent is $L$. major, with five genes ${ }^{10}$.

The existence of two distinct patient profiles (classic and severe) might be a consequence of several factors such as the infective strength level ${ }^{11}$, host immunosuppression ${ }^{8,12,13}$, and the genetic background of the host ${ }^{14,15}$, or it might be genetically determined by parasitic virulence factors ${ }^{16}$. The identification of genetic changes in the pathogenic target genes is a starting point in the search for virulence factors ${ }^{17}$. The term 'virulence factors' is used to describe proteins that are closely related to the severity of the disease or to genes encoding such peptides ${ }^{18,19}$. Genes such as A2, which causes a virulent phenotype when introduced in L. major ${ }^{20}$, or proteins such as the zinc metalloprotease glycoprotein (GP63) (leishmanolysin) which confer protection against lysis by the host ${ }^{10}$.

The NAGT gene of Leishmania is a single-copy gene with 1,401 base pairs per haploid genome ${ }^{21}$. The translated protein is a transmembrane protein of the endoplasmic reticulum (ER), identified as N-acetylglucosamine-1-phosphotransferase (NAGT), whose enzymatic action is the first step of $\mathrm{N}$-glycosylation ${ }^{22}$. Proteins that are extensively modified posttranslationally, including GP63, can become unstable because of mutations affecting $\mathrm{N}$-glycosylation, probably due to the decreased structural stability of leishmanolysin. This instability decreases the response against proteolytic degradation and causes the strain to be less virulent ${ }^{23}$.

Two genes that encode orthologues of human macrophage migration inhibitory factor (MIF) in the Leishmania genus have been described ${ }^{24-26}$. Human MIF is a major mediator of inflammation. This gene encodes a cytokine involved in cellmediated immunity, immunoregulation, and inflammation. MIF plays a role in the regulation of macrophage function in host defense through the suppression of the anti-inflammatory effects of glucocorticoids. The presence of MIF orthologues in other parasites (Eimeria sp., Trichinella sp., Plasmodium sp. and Brugia malay) is related to immune modulation ${ }^{27-29}$. Macrophage migration inhibitory factor gene-deficient mice (MIF -/-) are susceptible to L. major infection and develop significantly larger lesions and greater parasite burdens than do wild type resistant mice (MIF $+/+)^{30}$. Other studies report that recombinant MIF activates murine macrophages to kill L. major by increasing the levels of tumor necrosis factor alpha $(\mathrm{TNF}-\alpha)$ and nitric oxide production ${ }^{31,32}$.

The existence of two genes that exhibit significant sequence identity with mammalian MIF in the genome of Leishmania genus suggests a physiological role for macrophage migration inhibitory factor-like protein (MIF1 and MIF2) in the parasite life cycle and the establishment of these orthologues as virulence factors ${ }^{24-26}$. To date, the biological activities described for the L. major MIF orthologues include the induction of macrophage migration and an anti-apoptotic activity that could be responsible for the intracellular persistence of Leishmania in macrophages; thus, these MIF orthologues may be related to the clinical outcome ${ }^{25}$.

We investigated these three genes to determine whether the eventual presence of genetic heterogeneity such as single nucleotide polymorphism (SNPs) or indels in N-acetylglucosamine-1- phosphotransferase (NAGT) and in macrophage migration inhibitory factor-like protein (MIF1 and MIF2) of L. infantum could explain the severity of New World kala-azar.

\section{METHODS}

\section{Study design}

Two sets of patients participated in the study. For the NAGT study, 35 complication-free kala-azar patients and 33 kala-azar patients with complications (bleeding, opportunistic infections, sepsis or death) were included as shown in Table 1. For the study of the MIF orthologues, $43 \mathrm{~L}$. infantum isolates from other patients with uncomplicated kala-azar and 33 isolates from other patients with complications were analyzed for the presence of genetic polymorphisms (Table 1). The isolates were obtained from patients recruited from a reference hospital in Teresina, Brazil. The patients were referred from endemic areas of $L$. infantum infection in the neighboring States of Piauí and Maranhão. The clinical and laboratory diagnoses were confirmed by typical clinical presentations, such as fever, wasting, anemia and hepatosplenomegaly, in addition to reactive serology and the presence of the parasites in the bone marrow. The species identification was performed using monoclonal antibodies. The parasites were cultured in Novy-MacNeal-Nicolle (NNN) media with Schneider's insect medium (Sigma, St. Louis, United States of America) supplemented with fetal calf serum (Cultilab, Campinas, Brazil). The cultures were incubated at $28^{\circ} \mathrm{C}$ for five days before DNA extraction.

\section{DNA extraction, PCR and sequencing}

DNA was extracted with Chelex ${ }^{\circledR} 100$ resin (Bio-Rad, Hemel Hempstead, United Kingdom) and with a QIAamp ${ }^{\circledR}$ DNA mini kit (250) from Qiagen (Qiagen, Hilden, Germany). For the NAGT amplification, four primers were designed (L0, L2B, L3B and L5), based on the GenBank reference AF205934 (Table 2). For the MIF orthologue amplification, the primers were as follows: MIF1 forward 5'- ATGCCGGTCATTCAAACG-3' and MIF1 reverse 5'-TTAGAAGTTTGTGCCGTTCCA-3'; and MIF2 forward 5'-ATGCCATTTCTGCAGACGA-3' and MIF2 reverse 5'-TCAAAAGTTAGCGCCGTTC-3' (Table 2),

TABLE 1 - Rates of complications among selected patients* in the NAGT and MIF orthologues study.

\begin{tabular}{lccccc}
\hline & \multicolumn{2}{c}{ NAGT } & & \multicolumn{2}{c}{ MIF orthologues } \\
\cline { 2 - 3 } \cline { 6 - 7 } & $\mathrm{n}$ & $\%$ & & $\mathrm{n}$ & $\%$ \\
\hline No complications & 35 & 51.0 & & 43 & 57.0 \\
Complications** & 33 & 49.0 & & 33 & 43.0 \\
\hline Total & 68 & 100.0 & & 76 & 100.0 \\
\hline
\end{tabular}

NAGT: N-acetylglucosamine-1-phosphate transferase; MIF: macrophage migration inhibitory factor-like protein. *For each gene target, NAGT and MIF orthologues, were used different patients. **Complications: bleeding, opportunistic infections, sepsis or death. 
TABLE 2 - Primers for NAGT, MIF1, and MIF2 PCR and sequencing.

\begin{tabular}{lcc}
\hline Name & Target & Primers \\
\hline L0 & NAGT & CACAGAAGCTCTTCCTCGACTC \\
L5 & NAGT & GTAGAGACGGTCCTCGTTTCAC \\
L2B & NAGT & CGACTGCGATCACAATGCTC \\
L3B & NAGT & GGCACAGCGTTGATCTACCT \\
MIF1_f & MIF1 & ATGCCGGTCATTCAAACG \\
MIF1_r & MIF1 & TTAGAAGTTTGTGCCGTTCCA \\
MIF2_f & MIF2 & ATGCCATTTCTGCAGACGA \\
MIF2_r & MIF2 & TCAAAAGTTAGCGCCGTTC \\
\hline
\end{tabular}

NAGT: N-acetylglucosamine-1-phosphate transferase; MIF: macrophage migration inhibitory factor-like protein; PCR: polymerase chain reaction.

designed based on the LinJ33.2090 [GenBank: XM_001468252] and LinJ33.2100 [GenBank: XM_001468253] genes of L. infantum. The polymerase chain reactions (PCR) were performed in $40 \mu \mathrm{l}$ of reaction mixture containing $3 \mathrm{mM} \mathrm{MgCl} 2$, $200 \mu \mathrm{M}$ dNTPs, $0,5 \mu \mathrm{M}$ each primer, and $0.04 \mathrm{U}$ Platinum Taq (Invitrogen, Life Technologies, Carlsbad, United States of America) in a Mastercycler gradient programmed for one cycle of $7 \mathrm{~min}$ at $94^{\circ} \mathrm{C}, 25$ cycles of $1 \mathrm{~min}$ at $94^{\circ} \mathrm{C}, 1 \mathrm{~min}$ at $55^{\circ} \mathrm{C}$ (NAGT), $67.9^{\circ} \mathrm{C}$ (MIF1) or $61^{\circ} \mathrm{C}(\mathrm{MIF} 2)$ and $1.5 \mathrm{~min}$ at $72^{\circ} \mathrm{C}$, with a final extension at $72^{\circ} \mathrm{C}$ for $10 \mathrm{~min}$. Five microliters of the PCR product was electrophoresed in $5.7-\mathrm{V} . \mathrm{cm}^{-1}$ in $1 \%$ agarose gel in the presence of GelRed ${ }^{\circledR}$ and visualized under UV light.

The DNA strands were sequenced using ABI 3730XLs for the NAGT gene and MegaBACE 1,000 for the MIF orthologues at least six times in both orientations. The nucleotide sequences were assembled using the CAP3 Sequence Assembling Program $^{33}$ to generate a consensus sequence. The nucleotide sequences were aligned using Clustal W software ${ }^{34}$.

\section{Phylogenetic analyses}

Multiple sequence alignments were performed using Clustal $\mathrm{W}^{34}$ in Molecular Evolutionary Genetics Analysis Mega $5^{35}$. To calculate the evolutionary distances, phylogenetic trees were constructed by the maximum-likelihood estimation method, and the tree topologies were evaluated using Mega $5^{35}$. All 68 NAGT sequences were selected, with Leishmania infantum NAGT [GenBank: AF205934], L. infantum NAGT var. 2 [GenBank: DQ836147], L. infantum var. 4 [GenBank: DQ836148], L. infantum NAGT var 7 [GenBank: DQ836149], Leishmania donovani [GenBank: DQ836150], Leishmania major [GenBank: AF205930.1], Leishmania tropica [GenBank: AF291678.1], Leishmania mexicana [GenBank: M96635.1], Trypanosoma cruzi [GenBank: XM_807416.1], and Leishmania infantum JPCM5 chromosome 36: $1589082 \mathrm{bp}-1590482 \mathrm{bp}$. Another phylogenetic tree was constructed with the identical settings in the presence of one representative sequence of the sequences generated in this work.
For the MIF orthologues, neighbor-joining phylogenetic trees were constructed using the nucleotide and amino acid sequences of MIF1 (HLc1), MIF2 (HLc2), Lin33.2090, Lin33.2100, Lmj1740, Lmj1750, MIF-Lbra and MIF human; the nucleotide and amino acid percentages of identity among the Leishmania MIF orthologues were calculated using LALIGN software ${ }^{36}$.

\section{Ethical considerations}

This work is part of a research project, Influence of the Leishmania chagasi genotype over the visceral leishmaniasis pathology, which is registered by the National Council of Research Ethics (CONEP) under 0116.045.203-05 and was approved by the National Council of Ethics (CEP - Conselho Nacional de Ética) at the Federal University of Piauí in 1214-2005. Since then, Leishmania strains have been isolated from infected patients at the Natan Portella Tropical Diseases Institute (IDTNP - Instituto de Doenças Tropicais Natan Portella). At the time, the patients were advised regarding the research and signed the clarified free-consent form. This work has not presented harm to any patient because the Leishmania data used in the project were previously collected and stocked at $-70^{\circ} \mathrm{C}$ in liquid nitrogen. The patients were identified by numeric codes, which shielded their identities.

\section{RESULTS}

The nucleotide sequencing of the 144 amplicons (76 to the MIF orthologues and 68 to NAGT) revealed the absence of genetic variability of the NAGT, MIF1 and MIF2 genes among these analyzed isolates of $L$. infantum. The complete identity of all the MIF1 and MIF2 nucleotide sample sequences was observed with the $\operatorname{Lin} 33.2090$ and $\operatorname{Lin} 33.2100$ sequences of the L. infantum MIF orthologues (Figure 1).

In Figure 1, the cluster in which the MIF gene orthologues were located was divided into two distinct sub-clusters. The first cluster, located at the top of the tree, comprises the MIF1 (HLc1), Lin33.2090, Lmj1740 and LbrM33.V2.2010 genes. The second sub-cluster (lower) comprises the MIF2 (HLc2), Lin33.2100 and Lmj1750 genes. The LbrM33.V2.2030 gene from $L$. braziliensis appears to be more similar than the other MIF gene of Leishmania to the human MIF orthologues.

Similarly, the phylogenetic tree of NAGT indicates strong gene similarity independent of the strain or species (Figure 2). The tree with a representative sequence of all the sequences generated in this work (3928 contig 1) and other sequences from NCBI reveals that all of the NAGT sequences from the L. donovani complex are clustered together; however, L. mexicana, L. major and L. tropica each appear in different clusters.

\section{DIscussion}

The data indicate that the clinical variability and severity of kala-azar could not be genetically attributed to the studied genes. Factors such as other parasitic genes ${ }^{10,20,37}$ and host determinants including host genetics ${ }^{15}$, immune status ${ }^{8}$, and nutrition ${ }^{2}$ 

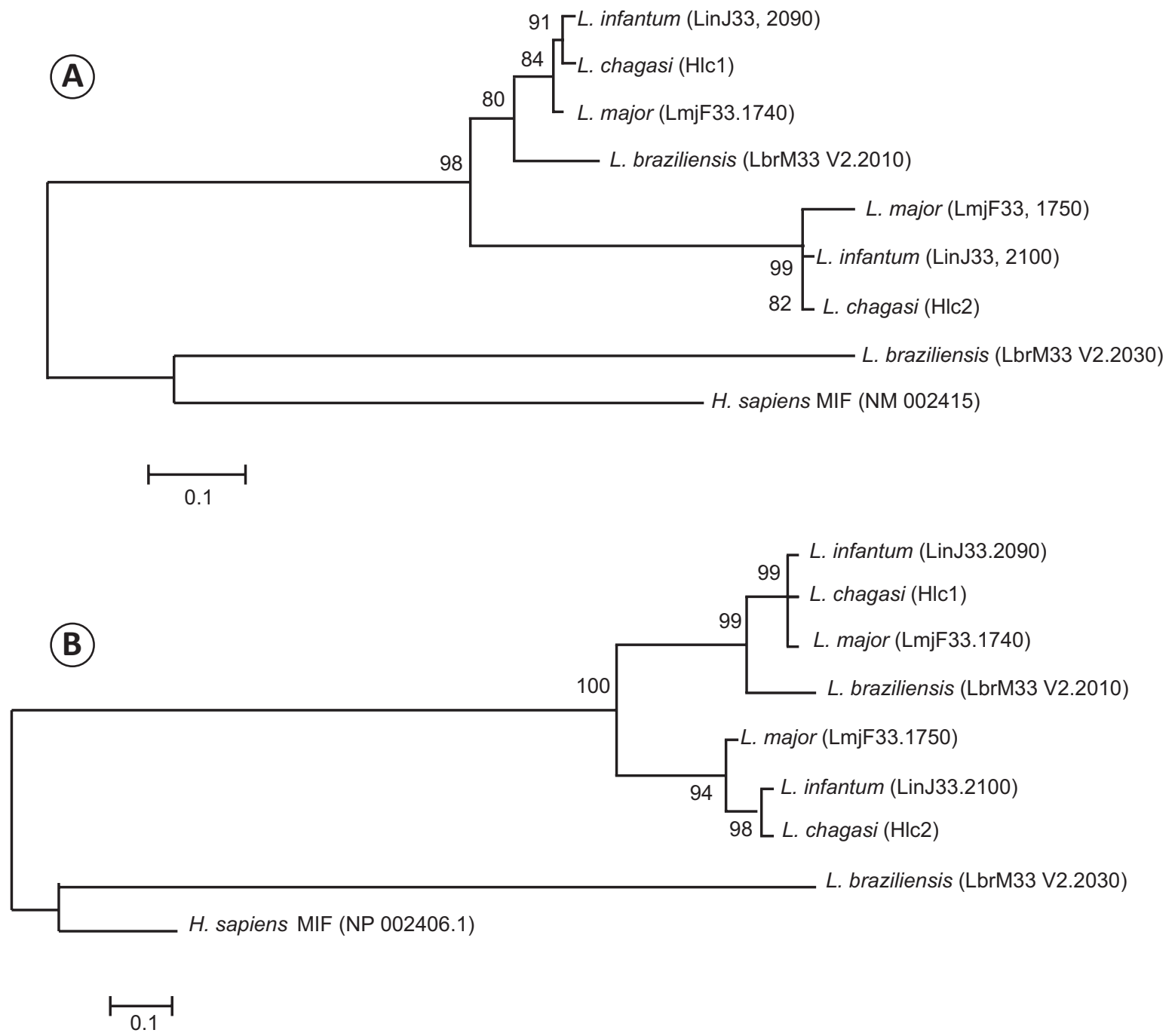

FIGURE 1 - Neighbor-joining phylogenetic tree for the MIF orthologues sequences. The phylogenetic trees were constructed using the sequences of nucleotides (A) and amino acids (B) of the MIF1 (HLc1), MIF2 (HLc2), Lin33.2090, Lin33.2100, Lmj1740, Lmj1750, MIF-Lbra and MIF human genes. L: Leishmania; H: Homo; MIF: macrophage migration inhibitory factor-like protein.

should be investigated ${ }^{10}$. Because of a likely polygenic influence on the phenotype of the disease, as suggested by the multiple molecular mechanisms associated with Leishmania survival in vertebrate hosts $^{38,39}$, and the crucial involvement of the host response, additional kala-azar patients and other genes should be observed before a definitive conclusion is reached on the role of virulence factors in the pathogenesis of severe kala-azar. This type of information could be critical for drug and vaccine development because the virulence factors might be targeted by specific drugs and may be related to the quality and magnitude of the host immune response ${ }^{40}$.

The data support the hypothesis that these genes are conserved in the genome of Leishmania, particularly those belonging to the $L$. donovani complex; all of the species that we used from this complex were arranged in a single cluster (Figure 2). We hypothesize the importance of NAGT for the visceralization of Leishmania as a predisposing factor for VL. After the genome sequencing of L. major, L. infantum and L. braziliensis, it was observed that only a minority of genes are species-specific genes ${ }^{10,41}$. Thus, the difference in the clinical presentation of visceral, cutaneous and mucocutaneous leishmaniasis could not be the result of significant genetic differences throughout the genome. The clinical presentations are likely a consequence of critical point mutations in a few genes or of post-transcriptional or post-translational mechanisms ${ }^{41}$. We could not exclude the possibility that the studied isolates were differed with respect to gene or protein expression levels, which might be related to the different clinical outcomes of kala-azar.

The high degree of conservation of these genes between the isolates and with the published sequence of $L$. infantum suggests that these genes might be critical for the survival of the parasites in the vertebrate host. This conclusion is drawn from the lack of genetic variability that could impair the 


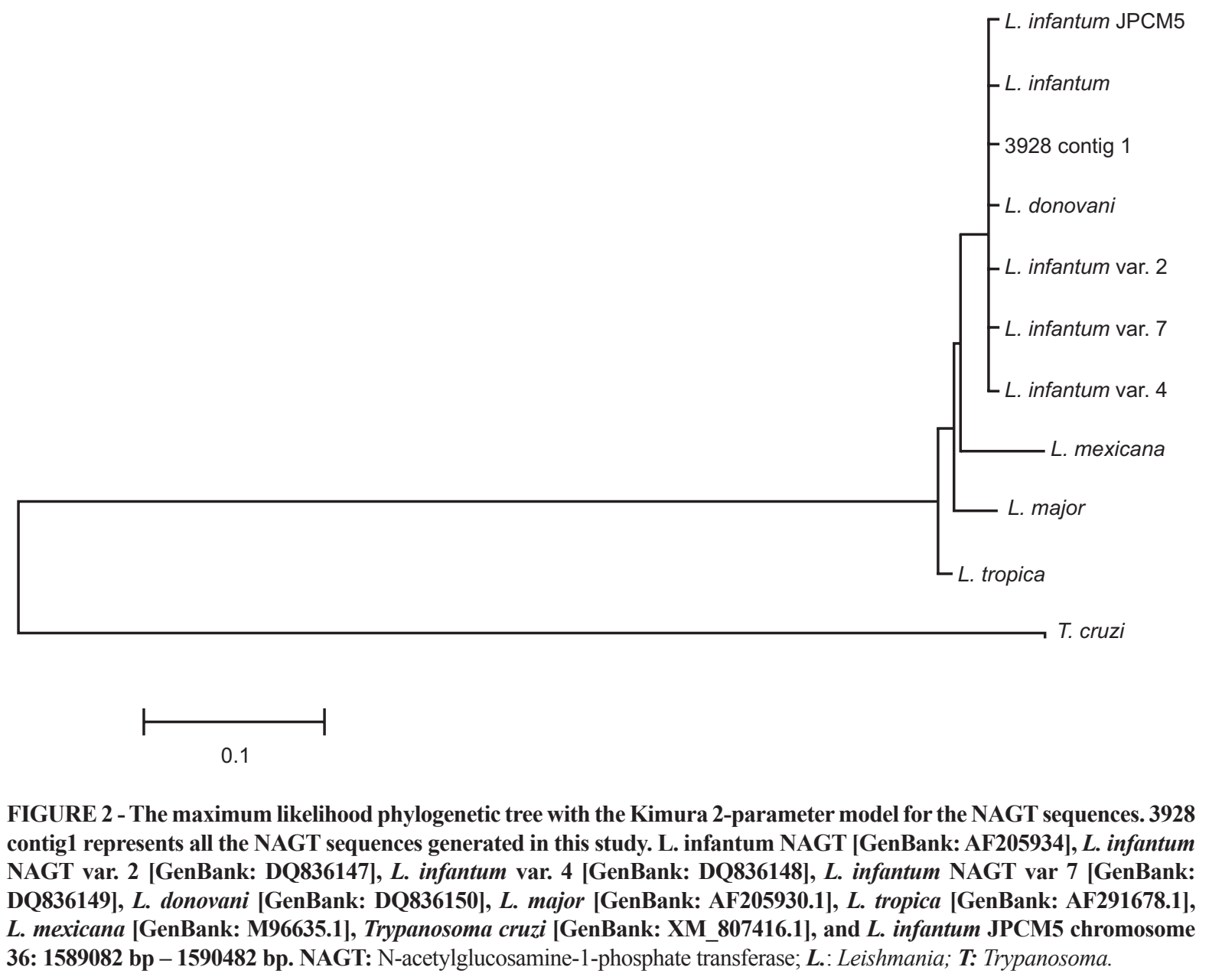

MIF orthologue functions involved with parasite survival inside macrophages or the structural stability of leishmanolysin from $\mathrm{N}$-glycosylation, in the case of NAGT.

The NAGT, MIF1 and MIF2 genes have a high sequence similarity to the L. infantum JPCM5 (NCBI) genes used for comparison and are not involved with the severity of kala-azar at the coding level, which suggests a critical role of these genes in the biological cycle of the parasite. Considering their conservation level in identical species, the products of these genes might be used in phylogenetic inferences or, eventually, for diagnostic purposes or second-generation vaccine development.

\section{ACKNOWLEDGMENTS}

We thank Dr. Guilherme Corrêa de Oliveira for providing the sequencing platform as well as Elisa Cupolillo and Carlos Henrique M. da Silva from FIOCRUZ for providing the standard strain of L. infantum L0579.

\section{CONFLICT OF INTEREST}

The authors declare that there is no conflict of interest.

\section{FINANCIAL SUPPORT}

This study received financial support from the Coordination of Training of Higher Education Graduate Foundation (CAPES) and the National Council of Technological and Scientific Development (CNPq).

\section{REFERENCES}

1. Alves WA. Leishmaniose visceral americana: situação atual no Brasil Leishmaniasis: current situation in Brazil. World Health 2009; 6:25-29.

2. Alvar J, Yactayo S, Bern C. Leishmaniasis and poverty. Trends Parasitol 2006; 22:552-557.

3. World Health Organization. Control of the leishmaniases. World Health Organ Tech Rep Ser 2010; xii-xiii:1-186.

4. Badaró R, Jones TC, Lorenço R, Cerf BJ, Sampaio D, Carvalho EM, et al. A prospective study of visceral leishmaniasis in an endemic area of Brazil. J Infect Dis 1986; 154:639-649.

5. Evans TG, Teixeira MJ, McAuliffe IT, Vasconcelos I, Vasconcelos AW, Sousa AA, et al. Epidemiology of visceral leishmaniasis in northeast Brazil. J Infect Dis 1992; 166:1124-1132.

6. Werneck GL, Rodrigues L, Santos MV, Araújo IB, Moura LS, Lima SS, et al. The burden of Leishmania chagasi infection during an urban outbreak of visceral leishmaniasis in Brazil. Acta Trop 2002; 83:13-18. 
7. World Health Organization. Special Programme for Research and Training in Tropical Diseases (TDR). Visceral leishmaniasis rapid diagnostic test performance. World Health Organization; 2011.

8. Costa CHN, Werneck GL, Costa DL, Holanda TA, Aguiar GB, Carvalho AS, et al. Is severe visceral leishmaniasis a systemic inflammatory response syndrome? Rev Soc Bras Med Trop 2010; 43:386-392.

9. Zhang J, Wheeler D, Yakub I, Wei S, Sood R, Rowe W, et al. SNPdetector: a software tool for sensitive and accurate SNP detection. PLoS Comput Biol 2005; 1:e53.

10. Deborah F. Smith, Christopher S. Peacock and AKC: Comparative genomics: From genotype to disease phenotype in the leishmaniases. Int J Parasitol 2007; 37:1173-1186.

11. Kimblin N, Peters N, Debrabant A, Secundino N, Egen J, Lawyer P, et al. Quantification of the infectious dose of Leishmania major transmitted to the skin by single sand flies. PNAS 2008; 105:10125-10130.

12. Ter Horst R, Collin SM, Ritmeijer K, Bogale A, Davidson RN. Concordant HIV infection and visceral leishmaniasis in Ethiopia: the influence of antiretroviral treatment and other factors on outcome. Clin Infect Dis 2008; 46:1702-1709.

13. Olivier M, Badaró R, Medrano FJ, Moreno J. The pathogenesis of Leishmania/HIV co-infection: cellular and immunological mechanisms. Ann Trop Med Parasitol 2003; 97 (suppl I):79-98.

14. Lipoldová M, Demant P. Genetic susceptibility to infectious disease: lessons from mouse models of leishmaniasis. Nat Rev Genet 2006; 7:294-305

15. Alonso DP, Ferreira AF, Ribolla PE, Miranda Santos IK, Socorro Pires e Cruz M, Aécio de Carvalho F, et al. Genotypes of the mannan-binding lectin gene and susceptibility to visceral leishmaniasis and clinical complications. J Infect Dis 2007; 195:1212-1217.

16. Khamesipour A, Rafati S, Davoudi N, Maboudi F, Modabber F. Leishmaniasis vaccine candidates for development: A global overview. Indian J Med Res 2006; 123:423-438.

17. Samaranayake TN, Fernando SD, Dissanayake VHW. Candidate gene study of susceptibility to cutaneous leishmaniasis in Sri Lanka. Trop Med Int Health 2010; 15:632-638.

18. Burgos JM, Risso MG, Brenière SF, Barnabé C, Campetella O, Leguizamón MS. Differential distribution of genes encoding the virulence factor trans-sialidase along Trypanosoma cruzi discrete typing units. PLoS One 2013; 8:e58967.

19. Lambertz U, Silverman JM, Nandan D, McMaster WR, Clos J, Foster LJ, et al. Secreted virulence factors and immune evasion in visceral leishmaniasis. J Leukoc Biol 2012; 91:887-899.

20. Zhang W-W, Mendez S, Ghosh A, Myler P, Ivens A, Clos J, et al. Comparison of the A2 gene locus in Leishmania donovani and Leishmania major and its control over cutaneous infection. J Biol Chem 2003; 278:35508-35515.

21. Akman L, Aksu HS, Wang RQ, Ozensoy S, Ozbel Y, Alkan Z, et al. Multi-site DNA polymorphism analyses of Leishmania isolates define their genotypes predicting clinical epidemiology of leishmaniasis in a specific region. J Eukaryot Microbiol 2000; 47:545-554.

22. Waki K, Dutta S, Ray D, Kolli BK, Akman L, Kawazu S-I, et al. Transmembrane molecules for phylogenetic analyses of pathogenic protists: Leishmania-specific informative sites in hydrophilic loops of trans-endoplasmic reticulum $\mathrm{N}$-acetylglucosamine-1-phosphate transferase. Eukaryot Cell 2007; 6:198-210.
23. Mcgwire BS, Chang K, Stability I, Exit E. Posttranslational regulation of a Leishmania HEXXH. J Biol Chem 1996; 271:7903-7909.

24. Peacock CS, Seeger K, Harris D, Murphy L, Ruiz JC, Quail M, et al. Comparative genomic analysis of three Leishmania species that cause diverse human disease. Nat Genet 2007; 39:839-847.

25. Richardson JM, Morrison LS, Bland ND, Bruce S, Coombs GH, Mottram JC, et al. Structures of Leishmania major orthologues of macrophage migration inhibitory factor. Biochem Biophys Res Commun 2009; 380:442-448

26. Kamir D, Zierow S, Leng L, Cho Y, Diaz Y, Griffith J, et al. A Leishmania ortholog of macrophage migration inhibitory factor modulates host macrophage responses. J Immunol 2008; 180:8250-8261.

27. Vermeire JJ, Cho Y, Lolis E, Bucala R, Cappello M. Orthologs of macrophage migration inhibitory factor from parasitic nematodes. Trends Parasitol 2008; 24:355-363.

28. Falcone FH, Loke P, Zang X, MacDonald S, Maizels RM, Allen JE. A Brugia malayi homolog of macrophage migration inhibitory factor reveals an important link between macrophages and eosinophil recruitment during nematode infection. J Immunol 2001; 167:5348-5354.

29. Cordery DV, Kishore U, Kyes S, Shafi MJ, Watkins KR, Williams TN, et al. Characterization of a Plasmodium falciparum macrophagemigration inhibitory factor homologue. J Infect Dis 2007; 195:905-912.

30. Satoskar AR, Bozza M, Rodriguez Sosa M, Lin G, David JR. Migrationinhibitory factor gene-deficient mice are susceptible to cutaneous Leishmania major infection. Infect Immun 2001; 69:906-911.

31. Jüttner S, Bernhagen J, Metz CN, Röllinghoff M, Bucala R, Gessner A. Migration inhibitory factor induces killing of Leishmania major by macrophages: dependence on reactive nitrogen intermediates and endogenous TNF-alpha. J Immunol 1998; 161:2383-2390.

32. Weiser WY, Temple PA, Witek-Giannotti JS, Remold HG, Clark SC, David JR. Molecular cloning of a cDNA encoding a human macrophage migration inhibitory factor. Proc Natl Acad Sci USA 1989; 86:7522-7526.

33. Huang X, Madan A. CAP3: a DNA sequence assembly program. Genome Res 1999; 9:868-877.

34. Larkin MA, Blackshields G, Brown NP, Chenna R, McGettigan PA, McWilliam H, et al. Bioinformatics 2007; 23:2947-2948.

35. Tamura K, Peterson D, Peterson N, Stecher G, Nei M, Kumar S. MEGA5: Molecular Evolutionary Genetics Analysis using Maximum Likelihood, Evolutionary Distance, and Maximum Parsimony Methods. Mol Biol Evol 2011; 28:2731-2739.

36. Pearson W. LALIGN-find multiple matching subsegments in two sequences. 2009. Available at: http://www.ch.embnet.org/software/ LALIGN_form.html/.

37. McCall L-I, Matlashewski G. Involvement of the Leishmania donovani virulence factor A2 in protection against heat and oxidative stress. Exp Parasitol 2012; 132:109-115.

38. Denkers EY, Butcher B. Sabotage and exploitation in macrophages parasitized by intracellular protozoans. Trends Parasitol 2005; 21:35-41.

39. Olivier M, Gregory DJ, Forget G. Subversion mechanisms by which Leishmania parasites can escape the host immune response: a signaling point of view. Society 2005; 18:293-305.

40. Flower DR. Bioinformatics for vaccinology. USA: Wiley; 2008.

41. Lynn M, McMaster WR. Leishmania: conserved evolution-diverse diseases. Trends Parasitol 2008; 24:103-105. 